\title{
The Need for Assessment and Reassessment of the Hospital Cancer Conference
}

\author{
Frederick L. Greene, MD \\ Department of General Surgery, Carolinas Medical Center, Charlotte, NC
}

For many years, a traditional event at hospitals throughout the United States has been the convening of intermittent conferences that serve as formats for discussing patient cancer management. These conferences have been generally associated with appropriate gustatory delights to further the attraction of these meetings. Generally a moderator has been responsible for developing the case list utilizing recent patients diagnosed or treated at the hospital. Because the desire to include the multidisciplinary concept (surgery, radiation oncology, and medical oncology) of cancer management has driven the case selection, a potpourri of case presentations has been the goal to create cross-cultural dialogue and encourage participation.

It has been my pleasure to attend many of these cancer conferences as a surveyor for the Accreditation (formally "Approvals") Program of the Commission on Cancer of the American College of Surgeons. These conferences held at small and medium-sized community hospitals, medical school-associated teaching hospitals, and National Cancer Institute-designated institutions are as diverse as the institutions involved. Some include crisp PowerPoint presentations of only three or four patients. Other formats include 10,15 , or more patients in an attempt to blend education along with clinical management.

My issue with most of these conferences is that they are based on a "show and tell" mentality rather than serving as treatment-planning conferences utilizing the expertise of the participating multidisciplinary experts. It has been a pleasure to see that more recently "organ-based" (breast, hepatopancreaticobiliary, gastrointestinal) and "diseasedbased"(sarcoma, melanoma) conferences have been

(C) Society of Surgical Oncology 2009

Published Online: 28 July 2009

F. L. Greene, MD

e-mail: frederick.greene@ carolinashealthcare.org created to serve as true treatment-planning conferences that facilitate decision making, especially in this era of neoadjuvant therapy. This is truly the format that should be supported - not the anachronistic approaches of the traditional hospital "tumor board."

In this issue of Annals, Wright et al. describe the prevalence of multidisciplinary cancer conferences (MCCs) in the province of Ontario, Canada, and analyze the barriers to the adoption of meaningful planning conferences. ${ }^{1}$ General surgeons throughout Ontario were queried about their perceptions regarding MCC implementation. In reply to a survey, a reasonable (44\%) response was obtained, which represented surgeons working in both community and academic institutions. Only 52\% of hospitals had MCCs, which were held over a variety of frequencies ranging from weekly to monthly. The majority of those surgeons responding had positive responses favoring the educational and collegial nature of these conferences.

The obvious lacuna in the study is the absence of any data that indicates that patients who are discussed have benefited from these multidisciplinary enclaves. Although published examples exist of cancer conference follow-up that assess adherence to management recommendations, these examples are few and the methodology to capture these data are problematic. ${ }^{2-4}$ Recent strategies by the American College of Surgeons Commission on Cancer have urged inclusion of published guidelines to serve as benchmarks of multidisciplinary care at $\mathrm{COC}$ accredited hospitals.

Aside from guideline overview, the ideal multidisciplinary cancer conference should include a meaningful discussion of treatment options supported by evidencedbase reports. Clinical staging should result from presentations of all relevant physical examination, imaging, laboratory, and pathological assessments. From these data, the clinical stage should be derived, which permits a logical discussion of all treatment options. Appropriate 
clinical trials should be reviewed to enhance patient care and trial accrual. Ideally these conferences should be organ- or disease-based and should incorporate all practitioners who have a stake in that particular patient's care. No treatment should be initiated before discussion in a multidisciplinary enclave.

Our Canadian brethren plan to use their study results to push for inclusion of MCCs in the appropriate hospitals in Ontario. The really challenging project is to craft a template that would ensure a meaningful treatment outcome for the patients presented. Although collegiality and education are laudable goals, the traditional MCC, absent of meaningful treatment planning, can no longer be our paradigm. The authors correctly invoke the importance of teleconferencing, especially from academic centers, and I personally believe this is a laudable goal.

Because one of the barriers to effective MCC in the Canadian study is lack of administrative support, we would do well to share this study with our administrative management teams. Quality management of our patients with cancer begins with the full support of our administrators, physicians, and all cancer care providers to maintain the quest for ideal cancer treatment.

\section{REFERENCES}

1. Wright FC, Lookhong N, Urbach D, et al. Multidisciplinary cancer conferences-identifying opportunities to promote implementation. Ann Surg Oncol. 2009. DOI:10.1002/ejoc.200700567.

2. Petty J, Vetto J. Beyond doughnuts: tumor board recommendations influence patient care. J Ca Educ. 2002;17:97-100.

3. Gatliffe TA, Coleman RL. Tumor board: more than treatment planning - a 1-year prospective survey. J Ca Educ . 2008;23:235-7.

4. Newman EA, Guest AB, Helvie MA, et al. Changes in surgical management resulting from case review at a breast cancer multidisciplinary tumor board. Cancer 2006;107:2346-51. 